\title{
In Silico Molecular Modeling \& Docking of Phytochemical Compounds with Odorant Binding Proteins (OBP1) of Culex Quinquefasciatus.
}

\author{
J.Venkateshwara Rao
}

\begin{abstract}
Malaria, Dengue Fever, West Nile Encephalities, Sleeping Sickness, Leishmaniasis, Japanese encephalitis (JE) are the widest large range of diseases causing highest mortality to human beings at Global level and they belong to the group Vector borne diseases (VBD). It is estimated that more than one million deaths were happening every year mostly in tropical regions of South America, Africa \& Asia due to these vectors i.e., mosquitoes and mites, which are main disease transporting vectors from one host to another. A remarkable effort has been made to develop various types of insecticides and insect repellents. To control VBD, a bio-defense strategy methods have been employed which were found to be more costly and labour intensive, recurring and time consuming. A new class of repellents were made based on structure based rational approaches of ligand molecules based binding efficiencies with Odorant binding proteins (OBPs) or other olfaction inhibitory compounds with attractive characteristics.
\end{abstract}

But, with very limited knowledge available to screen photo-chemical compounds to design novel mosquito repellents employing a very high-throughput Insilco computational biology methods. Therefore, in this context, we attempted to screen out 3 phytochemicals from different plants exhibiting mosquito repellent activities reported from published literature and various public domains \& molecular docking studies, aiming at the Odorant Binding Proteins of Culex quinquefasciatus. The $\mathrm{N}$, $N$-Diethyl-m-toluamide (DEET) was widely used mosquito repellent chemical chosen as standard reference to validate the binding affinities and specificities of selected compounds aligned with Odoront Binding Proteins. A total of such 50 compounds including DEET were docked against the active site of $O B T$ models or Crystal Structures using AutoDock. Among 5 phytochemical compounds, sum of 3 compounds have resulted in high affinity binding energies \& high no of hydrogen-bonds as compared to standard reference of DEET. Among the selected Citronellol, Saponin, and Azadirachtin, are showing the highest docking scores which secure to develop more effective and safer mosquito repellents in future prospect.

Revised Manuscript Received on December 30, 2019.

* Correspondence Author

Dr.J.Venkateshwara Rao, Asst.Professor, Department of Zoology, University College of Science, Osmania University, Hyderabad, Telangana, India. Email: venbio@gmail.com.

(C) The Authors. Published by Blue Eyes Intelligence Engineering and Sciences Publication (BEIESP). This is an open access article under the CC BY-NC-ND license (http://creativecommons.org/licenses/by-nc-nd/4.0/)
Key words: Odorant-binding protein, Phyto-chemicals, DEET, Azadirachtin.

\section{INTRODUCTION}

Mosquito repellents of variant forms ranging from extraction from medicinal plants, herbs, aromatic extracts and synthetic oils were widely used across the globe to protect human beings from mosquito borne diseases. To reduce man-mosquito interactions repellants can be used as a primary source, which can control the mosquito behavior, interruption of the usual olfactory reactions to diverse odor molecules. World Health Organization (WHO) has estimated that world climate change is main phenomenon which is enlarging mosquitoes habitat range, throwing up the threat of disease to millions of superfluous people every year ( WHO, 2009).

The broadening of diseases can be restricted either by taking control on the spreading number of disease causing vectors strictly or through avoiding the interface among the vector and host and which can be played as most essential step in prevention of the disease in the initial stages itself. The Culex quinquefasciatus mosquito, which is a vector for many pathogens like West Nile Virus, Zica virus, avian pox and protozoa like Plasmodium relictum, which causes bird malaria. It uses a series of olfactory signals that are coming out from human body odour or sweat to select human as their primary target food or blood meal. Prevention is always better than cure. Gorgeous advance is to prevent the transmission of above diseases by commotion of normal retorts to specific odorants that guide mosquito - human interactions, that relays upon the sensitivity of these definite odors inside the vector olfactory system which involves firm connection between odorant binding proteins (OBP's) and odoront receptors (Emma and Murphy, 2012). The OBP proteins are well distinguished by a unique 6 alpha-helical domains consisting of 6 highly conserved cysteine domains that are having highly distinctive disulphide bondages and that are in a range of $10-30 \mathrm{kDa}$ globular and are water soluble and compromised of large specific multi-gene family. It was observed that prospective insecticides or repellents take part in a vital position in the interruption of the spreading of mosquito borne diseases at the personal or at society level are produced by plants and herbs ( Patter, 2005). Recent studies have confirmed that in contrast to herbs, extracts from broad array of plants are more advantageous and successful(J.G. Millar, J.D. Chaney,1992). Amongst plant groups Cymbopogon, Ocimum and Eukalyptus are having with optimistic levels of essential oils and are used as repellents and are most cited in literature ( Venugopal and Gaddaguti, 2015). According to (TRECYDA, 2011), which is a traditional medicine literature, cananga (Canangium odoratum), patchouli (Pogostemon cablin), citronella (Cymbopogon nardus) and lemongrass (Cymbopogon citratus) are frequently used as insect repellents. 


\section{In Silico Molecular Modeling \& Docking of Phytochemical Compounds with Odorant Binding Proteins (OBP1) of Culex Quinquefasciatus.}

The current study primary aim is to investigate the possibility of searching out the repellent action of prospective natural compounds from a range of plant species against odoront binding proteins of Culex quinquefasciatus.

In this context, a sincere effort was made to realize the efficient mechanisms that rules the interactions of natural mosquito repellent compounds against odoront binding proteins of Culex quinquefasciatus.

\section{MATERIALS AND METHODS:}

\section{Retrieval of Mosquito Repellents from Phytochemical Literature Survey:}

For generations, plant based repellents have been used for individual defense measure against host- looking for mosquitoes. Ethnobotanical surveys are useful source of information for the development of natural repellents as much of knowledge is readily available about traditional plants. Active phyoto molecules were extensively searched by a thorough literature survey and suitable articles were retrieved based on repellent information from public websites by key word based search and expression based searching methods (T.O. Olagbemiro, 2004). From the literature collected about 8 different plants with important phytochemicals as ingredients and with repellent activity were selected. Artemisia monosperma, Ocimum tenuliflorum, Eugenia aromatic, Vertiveria zizanioides, Cymbopogon nardus, Hierochloe odorata, Nepeta cataria, Ocimum basilica etc.,

\section{Identification of Phytochemical Compounds:}

2D structures were obtained from a sum of 10 compounds which were identified as effective repellents and corresponding units were built using the PubChem database found at the website address:(http://pubchem.ncbi.nlm.nih.gov/).Using Discovery Studio Visualizaer 4.0 (DSV) the 2D coordinates were converted into 3D coordinates.

\section{Selection of Target Protein Molecule Modelling of target 3Dimentional OBPs Molecules:}

The main olfactory component system composed of insect Odorant binding proteins of insects bind to attract and as well as repellent odors. The transportation of odorants \& pheromone molecules to the olfactory receptors are recognized by odorant binding proteins and are the key proteins involved in transportation. Therefore, they are primarily identified as crucial target for improvement of new repellent molecules. Different kinds of crystal structures of OBS from various different kind of mosquitoes and insects have been identified and reported in the form of PDB structural files. But, unfortunately, no crystal structure of Culex quinquefasciatus were reported at RCSB databse in PDB format for Odornat binding proteins (OBPs). By using Computational Tools an attempt was made to built the 3-dimentional model of these OBPs proteins (Xu, et al.2019)

The OBPs (i.e., UniProt KB Accession: D2IGZ8 and B 5A5T7, full length primary sequences were downloaded from UniProtKB web site. By utilizing primary sequences the 3-D model of reliable theoretical model can be generated by using accurate computational modeling tools and employing the Comparative modeling methods ( G.Paluch, 2010)

Development of 3D structure of Odorant Binding Proteins from Culex quinquefasciatus.

A total of 7 OBPs crystal structures of were obtained as PDB files, a filter was employed for this purpose of extraction ( below $<=2.5$ Å resolution)

Heteroatoms \& water molecules along with substrates and small molecules were removed prior to crystallization, docking of these OBPs molecules. From the published literature the active site residues were identified for the purpose of docking studies.
DOCKING Active sites of OBP's with Selected Phytochemical Compounds:

Accurate molecular docking studies were conducted employing AutoDock Tools and AutoDock 4.2.6 in the process to investigate the accurate binding mechanisms of phytochemical compounds with the desired target OBP molecules. Hydrogen molecules were added to the polar atoms initially before the docking simulations and all the electric charges were being applied to each and every atom of the target molecule odour binding molecules. Non-Polar hydrogens, torsional angle of freedom, all rotating bonds were applied to the target molecule.

With the support of published literature, the docking stimulations were performed using variable grid dimensions covering of every one of putatative residues of the target molecule. A grid spacing of $0.375 \AA$ was performed underneath hybrid Lamarckian Genetic Algorithm with the no. of docking processes marked as high as 30 for molecular docking simulations.

From the available Autodock, the Lamarckian genetic algorithm is considered as one of the best known docking procedures and was implemented to perform the molecular docking studies. The following values were fixed for LGA: A max. No. of 2,50,000 energy evaluations, a max. No. of $27 \mathrm{~K}$ generations \& mutations and crossover rates of 0.02 and 0.8 respectively. Up to $2 \AA$ RMSD tolerance was used for clustering of final docked simulations. Each and every ligand docked conformations were placed into groups based on the bind energies and highest ranked clusters and conformations were examined for binding efficiencies.

\section{Docking the active site of Odorant Binding Proteins with} DEET:

In the current study, active sites of OBP were docked with most widely used repellent i.e., DEET with identical grid and docking parameters were utilized for docking with the selected phytochemicals which were listed as above. The binding efficiencies for selected phytochemical with OBPs of A.stephensi and A. gambiae were compared with standard DEET as reference to gauge the accuracy of docking results.

\section{Docking Results Analysis:}

Using LigPlot+ v. 1.4 and PyMol, the non-bonded interfaces including hydrogen bondings, hydrophobic and electrostatic relations between docked compounds and target Odorant binding proteins, results were analyzed. Using the Visualizer software , Discovery Studio 4.0, The specified analysis of residues involving in the contact between ligands and protein was identified and results were analyzed.

\section{RESULTS:}

\section{Literature survey on plant based compounds as mosquito repellent}

Initially around more than 100 published literatures (mostly from ethno botanical / ethno pharmacological surveys) on plant based compounds as repellent were collected from public domains. All total, a set of 10 phyto-chemical compounds were screened from 5 common plants used as mosquito repellant (as summarized in Table 1). 
Table 1: List of the plants along with the details of plants parts used as mosquito repellent obtained from primary literature survey.

\begin{tabular}{|c|c|c|c|c|}
\hline S.No & $\begin{array}{l}\text { Name of the } \\
\text { plant }\end{array}$ & Location & Plant Part & Reference \\
\hline 1 & $\begin{array}{l}\text { Ocimum } \\
\text { basilicum }\end{array}$ & India & $\begin{array}{l}\text { Leaves and } \\
\text { Shoot }\end{array}$ & $\begin{array}{l}\text { Pavela et.al, } \\
2014\end{array}$ \\
\hline 2 & $\begin{array}{l}\text { Citronella } \\
\text { muconata }\end{array}$ & India & Shoot & $\begin{array}{l}\text { Prajapathi } \\
\text { et.al, } 2005\end{array}$ \\
\hline 3 & $\begin{array}{l}\text { Azadirachta } \\
\text { indica }\end{array}$ & India & Leaves & $\begin{array}{ll}\text { Misra et.al } \\
1995\end{array}$ \\
\hline 4 & $\begin{array}{l}\text { Cymbopogon } \\
\text { nardus }\end{array}$ & India & $\begin{array}{l}\text { Leaves and } \\
\text { Stem }\end{array}$ & $\begin{array}{l}\text { LS Wei et.al., } \\
2005\end{array}$ \\
\hline 5 & $\begin{array}{l}\text { Hierochloe } \\
\text { odorata }\end{array}$ & India & Leaves & $\begin{array}{l}\text { Gregonis et.al, } \\
2005\end{array}$ \\
\hline
\end{tabular}

2. Mining of PhytoChemical Compounds from PubChem:

\begin{tabular}{|ll|l|l|}
\hline S.No & $\begin{array}{l}\text { Name of the } \\
\text { Compound }\end{array}$ & $\begin{array}{l}\text { Compound } \\
\text { ID (CID) }\end{array}$ & $\begin{array}{l}\text { Molecular } \\
\text { Formula }\end{array}$ \\
\hline 1 & Citronella & 7794 & $\mathrm{C}_{10} \mathrm{H}_{18} \mathrm{O}$ \\
\hline 2 & Citral & 638011 & $\mathrm{C}_{10} \mathrm{H}_{16} \mathrm{O}$ \\
3 & Myrcene & 31253 & $\mathrm{C}_{10} \mathrm{H}_{16}$ \\
\hline 4 & Azadirachtin & 5281303 & $\mathrm{C}_{35} \mathrm{H}_{44} \mathrm{O}_{16}$ \\
5 & Saponin & 198016 & $\mathrm{C}_{58} \mathrm{H}_{94} \mathrm{O}_{2}$ \\
\hline
\end{tabular}

Phytochemical compounds are extracted from Chembase and NCBI sites. Their two dimentional and three dimentional strctures were downloaded from Zinc chemical databases. Two dimensional structures were converted to three dimentional structures using PyRX software.

\section{Target odorant binding protein (OBPs) from Culex} quinquefasciatus.

\begin{tabular}{|c|c|c|c|c|c|c|c|}
\hline OBP NAME & ACCNO & A.ACIDS & $M . W$ & $p l$ & CYSTEINES & SIGNAL & EVALUE \\
\hline CquiOBP1 & AF 468212 & $149 / 125$ & 14.486 & 5.52 & $26 / 3 / 37 / 8 / 8$ & 98,9 & $(10-19)$ \\
\hline CquiOBP2" & FJ947084 & $146 / 124$ & 14.811 & 5.33 & $26 / 3 / 37 / 8 / 8$ & 99.9 & $(4 \mathrm{e} \cdot 23)$ \\
\hline CquiOBP3* & FJ947085 & $147 / 129$ & 14.539 & 5.42 & $27 / 3 / 37 / 8 / 8 / 111$ & 95,9 & $(8 \mathrm{e}-20)$ \\
\hline CquiOBP4' & FJ947086 & $150 / 132$ & 15.477 & 5.35 & $27 / 27 / 3 / 38 / 8 / 8$ & 99,9 & $(5 e-14)$ \\
\hline CquiOBP5* & FJ947087 & $143 / 128$ & 14.873 & 5.01 & $28 / 3 / 38 / 9 / 8$ & 87,1 & $(4 e-14)$ \\
\hline CquiOBPG ${ }^{*}$ & FJ947088 & $146 / 125$ & 13.844 & 8.22 & $28 / 3 / 41 / 10 / 8$ & 99,7 & $(5 e-17)$ \\
\hline CquioBP? & EU816362 & $146 / 126$ & 14.162 & 5.25 & $13 / 12 / 3 / 399 / 8 / 8 / 11$ & 1,2 & $(5 e-14)$ \\
\hline CquiOBP8' & FJ947089 & $144 / 121$ & 13.216 & 8.54 & $26 / 3 / 40 / 10 / 8$ & 99,8 & (1e-12) \\
\hline CquiOBPg* & FJ947090 & $147 / 123$ & 13.826 & 6.51 & $28 / 3 / 40 / 10 / 8$ & 99,9 & $(7 \mathrm{e}-14)$ \\
\hline CquioBP10 & XP_001864761 & 132 & 14.734 & 82 & $26 / 3 / 40 / 10 / 8$ & NO & $(40-13)$ \\
\hline
\end{tabular}

\begin{tabular}{|c|c|c|c|c|}
\hline CquioBP1 & CquioBP2 & CquioBP3 & CquioBP4 & CquioBP5 \\
\hline $\begin{array}{c}4 \\
\varepsilon^{2} y^{6}\end{array}$ & 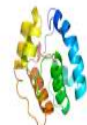 & $\begin{array}{l}56 \\
35 y \\
5 y\end{array}$ & की & $\begin{array}{r}5 \\
5 y^{4} \\
-52\end{array}$ \\
\hline CquiOBP7 & CquiOBP8 & CquioBp9 & CquioBP10 & CquioBP11 \\
\hline $\begin{array}{l}45 \\
5 y^{4}\end{array}$ & Sरदे & & & है? \\
\hline
\end{tabular}

The sequential information related to OBP proteins of Culex quniquefasciatus were extracted from NCBI websites. Their corresponding accession numbers are quoted in the above table. Corresponding models were developed using SPDBVIEWER software.

Modelled OBPs were prepared using PYMOL software by removing hetero atoms including hydrogens and small molecules along water of crystallization. From the literature reviews and docking studies of previous scientific reports active sites were identified.

\section{PHYTOCHeMicals USED FOR DOCKING WITH OBP} PROTEINS:

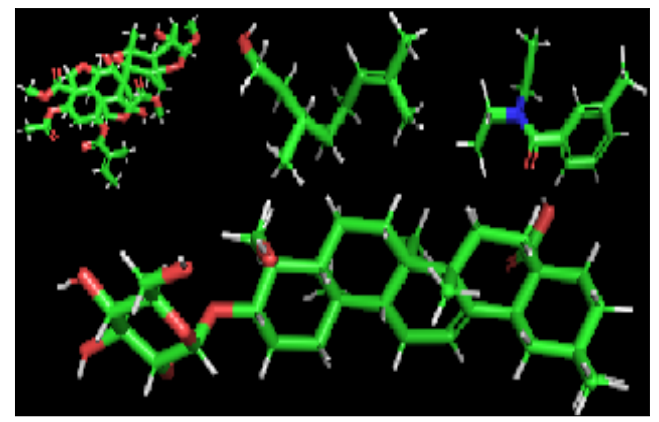

The chemical compounds with their 2D structures were extracted from Zinc Chemical Database or pubchem website at URL link address: http://pubchem.ncbi.nlm.nih.gov. The obtained ligands structures were then transferred to AUTODOCK recognizable format for next level studies.

Phytochemicals were selected for docking procedures were displayed in clock wise manner DEET, SAPONIN, CITRONELLA, AZADIRACHTIN. They were displayed using PYMOL software.

\section{PHYTOCHEMICALS DURING DOCKING PROCESS WITH OBD PROTEINS.}

The 3 Dimensional representation of CquiOBP1 associated with MOP, which is an ovi-position pheromone, is the first observed ligand bound structure of Culex OBP which gives close opportunity to look into binding pocket of Mosquito OBPs. CquiOBP1 manages to alter the EAG responses of MOP, Indole and Skatole confirming that the CquiOBP1 is crucial for recognition of these molecules ( Pelletier et al., 2010).

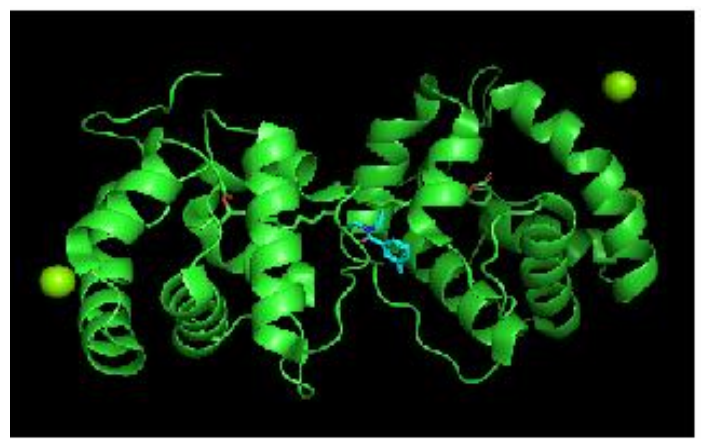

Culex OBP1 DOCKED WITH DEET

The CquiOBP1 ligand binding pocket was compared with other insect OBP structures and concluded that a central hollow space inside the protein covered by neighboring hydrophobic residues. In between the helix 4 and helix 5 the major part of the ligand molecules were associated in the hydrophobic tunnel. 
In Silico Molecular Modeling \& Docking of Phytochemical Compounds with Odorant Binding Proteins (OBP1) of Culex Quinquefasciatus.

\begin{tabular}{|c|c|c|c|}
\hline $\begin{array}{l}\mathrm{S} \\
\mathrm{N} \\
\mathrm{O}\end{array}$ & Compound & Protein & $\begin{array}{l}\text { Energy } \\
\text { Minimization } \\
\text { Kcal/Mol }\end{array}$ \\
\hline 1 & DEET & CuOBP1 & -4.7 \\
\hline 2 & Azadirachtin & CuOBP1 & -9.1 \\
\hline 3 & Citronellol & CuOBP1 & -4.8 \\
\hline 4 & Saponin & CuOBP1 & -6.2 \\
\hline
\end{tabular}

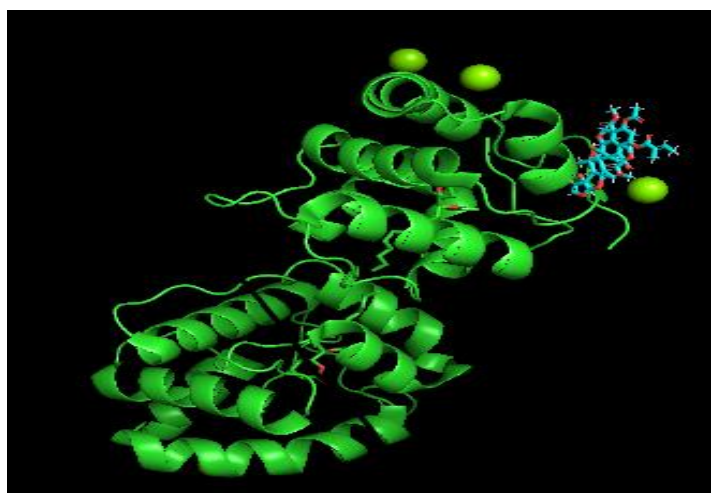

Culex OBP1 DOCKED WITH AZADIRCHTIN

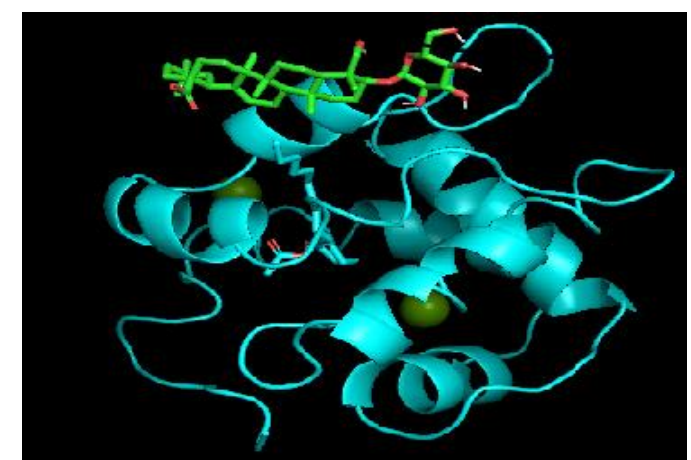

Culex OBP1 DOCKED WITH CITRONELLOL

OPLS-AA force field parameters were applied using Gromacs 4.5.3 software and energy minimization was done. As per the requirements of the AUTODOCK program the energy minimization structural files were prepared and by employing ADT tools hydrogen and gasteiger charges were added up. All the three dimensional coordinates of ligand MOP were obtained by Pubchem database. Twelve rotatable bonds were detected in the ligand and were chosen to be preserved during modeling process. Docking parameters were optimized by using Lamarkian genetic algorithm search method with different combination of parameters for Autogrid to reproduce a resultant conformation identical to experimentally determined crystal structure.

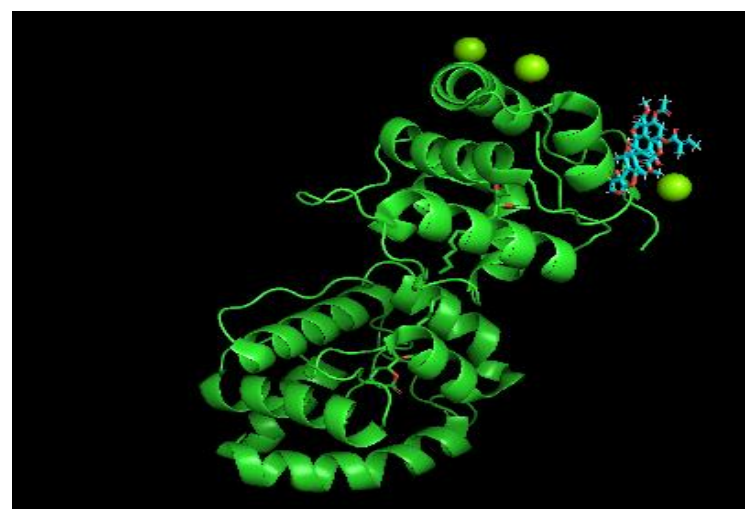

Culex OBP1 DOCKED WITH SAPONIN

\section{Energy Minimization:}

A grid box size of $70 * 55 * 68$ was employed with grid center of 32, 254; 37, 476; 37.56 which intimately identifies the binding portion of the OBPs. With $100 \mathrm{GA}$ runs and 25,00,000 evaluations with a population size of 150 Lamaackian genetic algorithm was employed and the ligands were treated as highly flexible units. Khodade et.al., 2007 had developed a parallel version of the program that used for cluster machine analysis was employed.

\section{DISCUSSION:}

The binding residues in helix4 for DEET binding pocket were identified as (Leu80, His77, Leu76, , Leu73) and binding residues in helix5 were identified (Ala88, Met91, Met89, Gly92) and helix6 (Trp144) and Leu96', Lys93', Arg94 and Leu96' where the (') refers to the dimer.

The results of our study revealed few potent compounds saponin, Azadirachtin, Citronellol etc. have high binding affinities and stronger hydrogen bond interactions with OBPs that DEET molecule which is widely used as mosquito repellent.

It was observed that Insect OBD proteins are the principle apparatus of olfactory system of all the insects that bind with all attractant or repellant odours and OBP1 protein of Culex in our studies have relvealed to show high binding affinities towards Citronellol, Saponin and Azadirachtin compounds. The determination of 3D structures and binding specificities of various plant based compounds with mosquito repellent activities could help us in devising much powerful repellants which are better than chemical compound DEET.

\section{CONCLUSION:}

The main advantage using essential oil as repellants against mosquitoes is they are accepted as safe when compared to chemically driven synthetic repellants and show less adverse affects on humans. The production and enhancement of mosquito repellents from plant sources is more productive to availability of plenty of ethnobotonical knowledge and databases. In the Current Study, Saponin, Azadirachtin, Citronellol have showed much more binding affinity than DEET which is a chemical based repellant. Atlast, from our studies it is revealed that much more potential natural repellants are available in plant databases and with the help of insilico studies we can sort out several compounds in shortest possible time and can produce efficient mosquito repellents than existing harmful synthetic chemical based mosquito repellants.

\section{REFERENCES:}

1. WHO (2009) Guidelines for Efficacy Testing of Mosquito Repellents for Human Skin

2. Emma and Murphy, (2012) “A novel mechanism of ligand binding and release in the odorant binding protein 20 from the malaria mosquito Anopheles gambiae." Protein science : a publication of the Protein Society vol. 22,1 (2013): 11-21.

3. Venugopal and Gaddaguti,( 2015). "Pharmacognostic and Preliminary Phytochemical Evaluation of Ocimum basilicum L . var . pilosum ( Willd . ) Benth . and O . tenuiflorum var . CIM-AYU." (2015).

4. TRECYDA, (2011) Mosquito OBD compounds 157, 136-144

5. J. Pelletier, D.T. Hughes, C.W. Luetje, W.S. Leal (2010) An odorant receptor from the southern house mosquito Culex pipiens quinquefasciatus sensitive to oviposition attractants

PLoS One, 5 (2010), Article e10090

6. P. Xu, Y.-M. Choo, Z. Chen, F.F. Zeng, K. Tan, T.-Y. Chen, .J. Cornel, N. Liu, W.S. LealOdorant inhibition in mosquito olfaction iScience, 19 (2019), pp. 25-38

7. J.G. Millar, J.D. Chaney, M.S. MullaIdentification of oviposition attractants for Culex quinquefasciatus from fermented Bermuda grass infusions Journal of the American Mosquito Control Association, 8 (1992), pp. 11-17 
8. T.O. Olagbemiro, M.A. Birkett, A.J. Mordue Luntz, J.A. PickettLaboratory and field responses of the mosquito, Culex quinquefasciatus, to plant-derived Culex spp . oviposition pheromone and the oviposition cue skatole Journal of Chemical Ecology, 30 (2004), pp. 965-976

9. G. Paluch, L. Bartholomay, J. CoatsMosquito repellents: a review of chemical structure diversity and olfaction Pest Management Science, 66 (2010), pp. 925-935

\section{AUTHORS PROFILE}

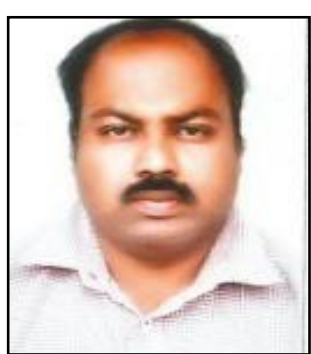

Dr. J.Venkateshwara Rao, M.Sc. B.Ed, M.Tech, Ph.D, Post Doc (USA), BOYSCAST Fellow, is an Asst.Professor in Dept of Zoology, Osmania University, joined in Osmania University in 2007. In 2009, he got Young Scientist and Investigator award from DBT under DBT-RGYI Scheme and in 2010 he got most prestigious DST BOYSCAST fellowship to pursue high end research in advanced areas. He went to Virginia Common Wealth University, Richmond and conducted research in Signal transduction cascades in smooth muscle cells of Gastrointestinal Muscles. 